Sharif University of Technology
Scientia Iranica

\title{
Security constrained optimal power flow in a power system based on energy storage system with high wind penetration
}

\author{
H. Ebrahimi ${ }^{a}$, M. Abapour ${ }^{a}$, B. Mohammadi Ivatloo ${ }^{a}$, and S. Golshannavaz ${ }^{b} *$ \\ a. Department of Electrical and Computer Engineering, University of Tabriz, Tabriz, Iran. \\ b. Department of Electrical Engineering, Urmia University, Urmia, Iran. \\ Received 25 June 2019; received in revised form 26 November 2019; accepted 1 June 2020
}

\author{
KEYWORDS \\ Renewable; \\ Uncertainty; \\ Security-Constrained \\ Optimal Power Flow \\ (SCOPF); \\ Energy Storage \\ System (ESS); \\ Security analysis.
}

\begin{abstract}
This study is focused on assessing the effect of Energy Storage System (ESS) on security improvement of power systems hosting remarkable renewable energy resources. To this end, the presence of ESS is suitably included in Security-Constrained Optimal Power Flow (SCOPF) model; the required technical amendments are hence considered. To launch a realistic model, ramping constraints of thermal units are also taken into account, which limit the generators from completely responding to power shortfalls. Considering the high penetration level of renewable generations, different scenarios of outages in transmission lines and generators are simulated to measure the Line Outage Distribution Factor (LODF) and Power Transfer Distribution Factor (PTDF). Also, in order to illustrate the economic impact of wind power generation curtailment and load shedding, Values of two penalty parameters of Wind Curtailment (VWC) and Value Of Loss of Load (VOLL) are considered in the model. Two test systems, including a PJM 5-bus system and an IEEE 24-bus RTS, are put under numerical studies to assess the possible impact of ESS on security improvement of the investigated systems. The obtained results are discussed in depth.
\end{abstract}

(C) 2022 Sharif University of Technology. All rights reserved.

\section{Introduction}

The soaring energy demand of power systems in different sectors including residential, commercial, and industrial suggests the need for further investment in power generation facilities. Meanwhile, the generationconsumption balance should be preserved with required reserve capacity. Beyond the conventional central generations that are mainly thermal units, it is now a common practice to deploy Distributed Generations

\footnotetext{
*. Corresponding author. Tel.: +984432775660 E-mail addresses: h.ebrahimi@ms.tabrizu.ac.ir (H. Ebrahimi); abapour@tabrizu.ac.ir (M. Abapour); bmohammadi@tabrizu.ac.ir (B. Mohammadi-Ivatloo); s.golshannavaz@urmia.ac.ir (S. Golshannavaz)
}

(DGs) to enhance the economic operation of power systems, increase the supply reliability [1], reduce power losses, suppress the pollutant emission, etc. Among these, renewable-based DGs such as wind turbines are recognized to be more environmentally-friendly resources [2]. In this context, most governments have utilized these resources in their power generation portfolio. However, the intrinsic uncertainties associated with these resources pose significant hurdles to power system operation, mainly in security analysis and perseverance. Contingency Analysis (CA) is a common task to assess the security level of the power system and consider preventive schedules.

Security-Constrained Optimal Power Flow (SCOPF) is a powerful tool for safe operation of power systems, especially when renewable generators such as wind turbine generators are connected to the 
system and they bring about uncertainty in the system [3]. SCOPF is an OPF problem considering some contingencies like generators and lines outages, against which the system should be secured. SCOPF is the incorporation of minimum cost and safe operation and security of the system [4-6]. To consider the security indices of a power system, there are some effective tools. One of these tools is calculation of linear sensitivity matrices. Won and Choi [7] measured two types of linear sensitivity matrices of control variables: voltage variations as well as reactive power generation and line flows. In this paper, both OPF and SCOPF solutions are obtained by LP and compared against each other. They argued that consideration of security constraints would increase operation costs, but any N-1 contingencies would not affect the system. Linear sensitivity factors including Power Transfer Distribution Factor (PTDF), Line Outage Distribution Factor (LODF), and Outage Transfer Distribution Factor (OTDF) are utilized to express the security constraints in the post-contingency state. Typically, SCOPF includes preventive and corrective types that differ from one another. In the Preventive SCOPF (PSCOPF), it is not allowed to reschedule control variables in the post-contingency state, except those with automatic responses associated with contingencies [8]. Moreover, an attempt is made to minimize cost function through only normal case control variables, which are feasible for both normal and contingent cases. This is while consideration of $\mathrm{C}$ contingencies makes the problem size to be approximately $\mathrm{C}+1$ time larger than the traditional OPF. Corrective SCOPF (CSCOPF) considers violation of some contingencies that the system can handle without damaging the devices. The total cost obtained by CSCOPF is often lower than the one by PSCOPF. However, the model requires some additional variables and perhaps a large number of reschedules for every contingency [9]. As it is explained in the referenced study [10], a secure system is defined at some levels, but the levels that SCOPF treats the system are as follows: Security level 1 is the system in which all loads are supplied, no operating limits are violated, and no limit violations occur in the event of a contingency. Security level 2 is the one that all loads are supplied, no operation limits are violated, and any violations caused by a contingency can be corrected through appropriate control actions without loss of load. The ideal operation condition for a system takes place when security level 1 is observed. However, security level 2 is more reasonable in terms of economics.

Evaluating the impact of renewables on power system security is of priority owing to their intrinsic uncertainties. Renewable generations like wind generation pose various uncertainties and this issue necessitates the need for security assessment of power system $[11,12]$. To this end, Prasanta et al. [13] considered a power system with high wind penetration and developed a Security-Constrained Unit Commitment (SCUC) model to assess the impact of Battery-ESS (BESS) units on the security of the system. To secure the system against the uncertainties of renewable generations, ESSs are one of the most effective tools. However, SCOPF for a system without ESSs needs a large model, which makes its solution time consuming [14]. Now, if the ESS is added to the system, the model will be very heavy and too much time is required to solve the problem [15]. A Benders Decomposition (BD) corresponding to a Mixed Integer Programming (MIP) was used to solve the SCUC problem in [13]. Prasanta et al. investigated the impact of BESSs on the security of systems with high penetration of wind power generations. It was illustrated that the BESSs charged at off-peak time and discharged during the peak time of the system; thus, the load curve of the system would be smoothened. Also, the presence of BESS in the system reduces the security cost. The SCUC model suffers from the lack of considering the transmission constraints of the power system. In [16], a model based on the AC-SCOPF was developed; however, the AC model's execution time was so excessive that it could not be utilized for operational purposes. An enhanced corrective SCOPF model was implemented in [17] to evaluate the impact of distributed BESS units on the security of a power system, but renewables were not considered. Among all security-concerned power system problems, it can be seen that contingencies to be studied are excessive, making it quite time consuming to exactly and comprehensively consider all of them.

Techniques that are utilized to reduce the number of noted contingencies include Contingency Filtering (CF) techniques. Platbrood et al. [18] proposed an iterative approach to solve the SCOPF problem. The process contains six major stages: (1) load flow, (2) SCOPF, (3) Security Analysis (SA), (4) CF, (5) PSCOPF, and (6) NC. The security analysis detects type of contingencies (overload or voltage collapse), the CF scheme is to identify binding constraints to be used in the problem solution, and Network Compression (NC) is used to reduce the complexity of the network model. The algorithm used here optimizes both active/reactive power flows together and treats discrete variables. Simab et al. [19] proposed an integrated method to rank the contingencies of the power system. As is clear, the impact of ESS presence on the security of the power system with high renewable generation penetration by means of SCOPF is still an interesting work to be done.

In this paper, a multi-period multi-stage MINLP DC-SCOPF model is developed to assess the impact of 
ESS units on the security of a power system with high wind generation penetration in a 24-hour time period. A 24-hour load curve and a 24-hour airflow pattern are employed to model the load and wind flow changes. In order to reduce the power losses of the transmission system, ESS units are sited at the buses where wind turbines lie [20]. In doing so, power curtailments of wind turbines are managed in this job [21]. In this work, the effect of ESS presence on security improvement of power systems hosting remarkable renewable energy resources is to be assessed. To do this, ESS presence is suitably included in the SCOPF model; the required technical amendments are hence considered. To have a realistic model, ramping constraints of thermal generation units are also taken into account that limit the generators from completely responding to power shortfalls. Considering a high penetration level of renewable generations, different scenarios of outages in transmission lines and generators are simulated to measure the Line Outage Distribution Factor (LODF) and Power Transfer Distribution Factor (PTDF). Also, in order to illustrate the economic impact of wind power generation curtailment and load shedding, two penalty parameters VWC and VOLL are considered in the model. Furthermore, the charging/discharging efficiencies of ESS units are considered, and to reduce the execution time of the model, a CF framework that selects only the binding contingencies is implemented. Finally, to illustrate the utilization performance of transmission lines and risk of operating the system, a Performance Index (PI) calculation is performed. In this paper, the main contributions can be listed in the following:

- Proposing secure operation of the power system with high wind penetration and presenting comprehensive evaluations of this task;

- Managing wind generation uncertainties by means of ESS units to ensure the security of the system;

- Reducing security cost of the system that consists of line outage and generation outage prohibition costs and consequently the operation cost;

- Significantly reducing contingencies against the system and enhancing the system security, in return.

\section{Model formulation}

Mathematical formulation of the SCOPF model in a system coordinated with wind generation and ESS is provided in this section. The model consists of an objective function and its related constraints. The objective function is the operation cost of the system. load flow equation and generation constraints of generators and line flow limits are the constraints of the conventional OPF problem. Security constraints for line outages and generator outages are considered. Also, wind generation constraints are added to the model. Furthermore, the constraints of ESS units' operation, including the State Of Charge (SOC) of units, maximum charge/discharge for each unit at each time interval and a constraint for asynchronous charge/discharge for each unit are added to the model.

\subsection{Objective function}

The objective function for this problem to be minimized consists of generating units' operation costs and load shedding penalty and the value of wind curtailment in each period.

$$
\begin{aligned}
O F= & \sum_{g, t}\left(a_{g}\left(P_{g, t}\right)^{2}+b_{g} P_{g, t}+c_{g}\right) \\
& +\sum_{i, t}\left(V O L L \times L S_{i, t}+V W C \times P_{i, t}^{w c}\right) .
\end{aligned}
$$

\subsection{OPF constraints}

The constraints of the conventional OPF problem for generating units and line flow limits as well as load shedding constraints and wind power generation are as follows:

$$
\begin{aligned}
& \left(\sum_{g \in \Omega_{G}^{i}} P_{g, t}\right)+L S_{i, t}+P_{i, t}^{w}-L_{i, t}-P_{i, t}^{c}+P_{i, t}^{d} \\
& \quad=\sum_{j \in \Omega_{l}^{i}} P_{i j, t}: \lambda_{i, t}, \\
& P_{i j, t}=\frac{\delta_{i, t}-\delta_{j, t}}{x_{i j}} \\
& -P_{i j}^{\max } \leq P_{i j, t} \leq P_{i j}^{\max }, \\
& P_{g}^{\min } \leq P_{g, t} \leq P_{g}^{\max }, \\
& P_{g, t}-P_{g, t-1} \leq R U_{g}, \\
& P_{g, t-1}-P_{g, t} \leq R D_{g}, \\
& 0 \leq L S_{i, t} \leq L_{i, t}, \\
& 0 \leq P_{i, t}^{w} \leq w_{i, t} \Lambda_{i}^{w}, \\
& P_{i, t}^{w c}=w_{i, t} \Lambda_{i}^{w}-P_{i, t}^{w} .
\end{aligned}
$$

Eq. (1) is the objective function of the problem. Eq. (2) is the load balance equation. Eq. (3) explains the power flow equation. Ineq. (4) is the thermal constraint of lines. Eqs. (5), (6), and (7) are the constraints of thermal generation units. Eq. (8) explains the load shedding constraint. Ineq. (9) illustrates the constraint of wind turbines generated active power, and Eq. (10) illustrates the amount of curtailed active power output of wind turbines. 


\subsection{Security constraints}

The main objective of this paper is to maximize the security of the system. To address the security of the system, security constraints must be added to the model of the power system.

To provide a mathematical base for security considerations, two security parameters, PTDF and LODF, which were calculated in [22], are used in this study. Also, the parameter used to calculate the participation amount of generators when one of them is excluded was calculated in [22]. However, according to the context of the book, the referenced authors considered that no generator would get to its maximum limit by increasing the production of each generator according to this parameter. Therefore, in this paper, the parameter is considered as a variable that takes into account the current generation of generators and then, calculates the participation factor.

$$
\begin{aligned}
& P T D F_{i, j, n m}=\frac{1}{x_{n m}}\left(\left(X_{n i}-X_{n j}\right)-\left(X_{m i}-X_{m j}\right)\right) \\
& L O D F_{i j, n m}=\frac{X_{i n}-X_{i m}-X_{j n}+X_{j m}}{x_{i j}\left(1-\frac{X_{n n}+X_{m m}-2 \times X_{n m}}{x_{n m}}\right)} \\
& \gamma_{i, j, t}=\frac{P_{g_{j}}^{\max }-P_{g_{j}, t}}{\sum_{\neq i}\left(P_{g_{k}}^{\max }-P_{g_{k}, t}\right)} \\
& -1.2 \times P_{i j}^{\max } \leq P_{i j, t}+P T D F_{\mathrm{n}, r e f, i j} \times P_{n, t}^{g} \\
& \quad-\sum_{m \neq n}\left[P T D F_{r e f, \mathrm{~m}, i j} \times \gamma_{m, n, t} \times P_{n, t}^{g}\right] \\
& \leq 1.2 \times P_{i j}^{\max }, \\
& -1.2 \times P_{i j}^{\max } \leq P_{i j, t}+L O D F_{i j, n m} \times P_{n m, t} \\
& \leq 1.2 \times P_{i j}^{\max } .
\end{aligned}
$$

Eqs. (11), (12), and (13) calculate PTDF, LODF, and participation factor, respectively. Ineqs. (14) and (15) are generation outage and line outage security constraints, respectively. According to the study of [23], the line flow limits for security constraints are considered as short-term emergency limits which are 10-20\% greater than normal line flow limits.

\subsection{ESS constraints}

Constraint (16) illustrates SOC content for each ESS unit:

$$
S O C_{i, t}=S O C_{i, t-1}+\left(P_{i, t}^{c} \eta_{c}-P_{i, t}^{d} / \eta_{d}\right) \Delta t,
$$

Ineqs. (17) and (18) are constraints on charge/ discharge power for each ESS unit, respectively:

$$
U_{i, t}^{c} P_{i, \min }^{c} \leq P_{i, t}^{c} \leq U_{i, t}^{c} P_{i, \max }^{c}
$$

$$
U_{i, t}^{d} P_{i, \min }^{d} \leq P_{i, t}^{d} \leq U_{i, t}^{d} P_{i, \max }^{d},
$$

Eq. (19) is to maintain the asynchronous charge / discharge in ESS units:

$$
\mathrm{U}_{i, t}^{c}+U_{i, t}^{d} \leq 1
$$

Ineq. (20) restricts the amount of SOC of each ESS unit:

$$
S O C_{i, \min } \leq S O C_{i, t} \leq S O C_{i, \max } .
$$

\subsection{Performance index}

In order to evaluate the performance of the system before and after the security considerations and also, with an increase in the load scale, a Performance Index (PI) was introduced in [24] as follows:

$$
P I_{M W}=\sum^{\Omega_{l}}\left(\frac{W_{i j}}{2 n}\right) *\left(\frac{P_{i j}}{P_{i j}^{\max }}\right)^{2 n} .
$$

\section{Solution method}

A three-stage procedure was applied to solve the SCOPF problem in a system coordinated with wind generation and ESS: (i) At the first stage, a conventional $\mathrm{OPF}$ is executed to calculate the optimal power flows, bus voltage angles, power outputs of thermal and wind turbine units, and the ESS units' charge/discharge amounts; (ii) At the second stage, a CA procedure is performed to take into account only the binding contingencies for the SCOPF problem. At this stage, the power flows calculated at the previous level are used; (iii) A SCOPF problem considering the binding contingencies acquired at the second stage is administered here.

According to the presence of binary variables related to ESS units' state of charge/discharge, the problem at each stage is solved as an MINLP problem. A GAMS code is executed for this problem. The SBB solver of GAMS program is utilized to solve the problem at stages (i) and (iii).

\section{Simulation results}

In order to evaluate the impact of ESS on the security of the system with high wind penetration, the wellknown PJM 5-bus test system and IEEE 24-bus RTS are employed. In order to evaluate the impact of ESS units' presence on the security of the system, the total operating cost for the $24-\mathrm{h}$ period from [25] and the number of binding contingencies against the system are compared in 4 scenarios. Scenario 1 does not consider both security constraints and ESS units' presence. Scenario 2 only considers the operation of the system with only security consideration. Scenario 3 
takes into account the implementation of ESS units but security constraints are not considered. In Scenario 4, both security constraints and employment of ESS units are considered.

\subsection{Case study 1: PJM 5-bus test system}

The system parameters are similar to those used in [26]. According to Figure 1, two wind generators and their relative ESS systems are added to buses 1 and 5 . The capacities of wind turbine generators at buses 1 and 5 are 125 and $250 \mathrm{MW}$, respectively. The maximum storable energies of the ESS units at buses 1 and 5 are 12.5 and $25 \mathrm{MWh}$, respectively. The ESS units charging/discharging power at each time interval is $0.2 *$ $S O C_{i}^{\max }$; charging efficiency $\left(\eta_{c}\right)$ for all ESS units is $95 \%$; and discharging efficiency $\left(\eta_{d}\right)$ is $90 \%$. There are two penalty factors in the model. The Value of Wind Curtailment (VWC) is set to $5 \$ / \mathrm{MW}$ and the Value of Loss of Load (VOLL) is set to $250 \$ / \mathrm{MW}$.

In this system, the total peak demand, the total installed thermal generation capacity, total installed wind turbine generation, and total installed ESS units are $900,1530,375$, and $37.5 \mathrm{MWh}$, respectively. Operation cost and number of affecting contingencies of each scenario are illustrated for the PJM 5-bus test system in Table 1.

As is clear, the number of binding contingencies is

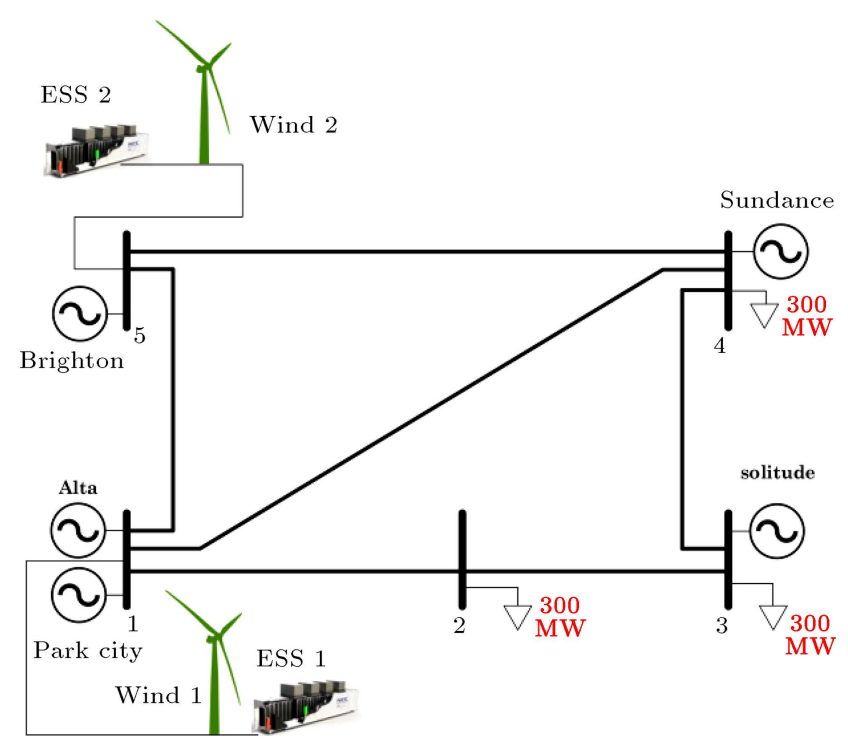

Figure 1. PJM 5-bus test system with wind generations and ESS units. reduced by $63 \%$, and the cost of security from Scenario 2 to Scenario 4 is reduced by $65.2308 \$$ for operation in a 24 -h period through the employment of ESS units. Security cost in scenarios 2 and 4 is $94619.908 \$$ and $94566.8956 \$$, respectively.

Figures 2 and 3 illustrate the SOC (MW) and total charge/discharge power (MW) of ESS units in the 24-h period of operation, respectively. The ESS units will charge when the gradient of load factor is around zero or when wind factor is high. They will also discharge when the gradient of load factor is highly positive or when the wind factor is low. In other words, ESS units will charge at the off-peak times of system demand and will discharge at peak times of system demand. In addition, each ESS unit charges when the related wind turbine is not curtailing and discharges when it is curtailing the generation. It is obvious that ESS 2 is not dispatched due to the absence of any load on the bus where ESS 2 lies and the cheapest generation unit at that bus.

Here, a performance index calculation for PJM 5 -bus test system is performed to see how security considerations affect the utilization performance for branches of the system. According to [24], the smaller the $P I_{M W}$ in one scenario, the better the performance of system branches utilization and the lower the risk

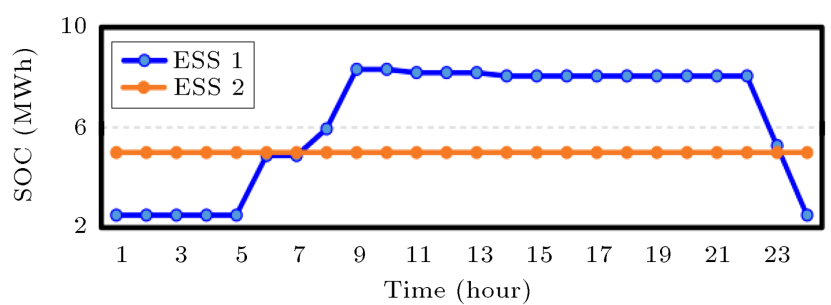

Figure 2. SOC of ESS units for PJM 5-bus test system.

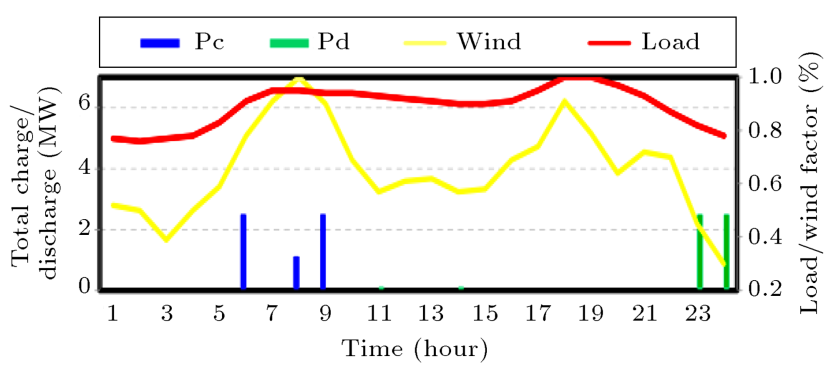

Figure 3. Total charge/discharge power of ESS units for the PJM 5-bus test system.

Table 1. PJM 5-bus test system operation cost and security comparison.

\begin{tabular}{ccc}
\hline Scenarios & Operation cost $(\$)$ & Number of contingencies \\
\hline Scenario 1 (no security + no ESS) & 175485.4209 & - \\
Scenario 2 (security + no ESS) & 270105.3289 & 667 \\
Scenario 3 (no security + ESS) & 175473.2025 & - \\
Scenario 4 (security + ESS) & 270040.0981 & 249 \\
\hline
\end{tabular}


Table 2. PJM 5-bus test system $P I_{M W}$ amount for each scenario.

\begin{tabular}{|c|c|c|c|c|}
\hline & $P I_{M W}($ sce. 1) & $P I_{M W}($ sce. 2) & $P I_{M W}($ sce. 3$)$ & $P I_{M W}$ (sce. 4$)$ \\
\hline 1 & 0.301506 & 0.012259 & 0.301506 & 0.012259 \\
\hline 2 & 4.448989 & 0.064956 & 4.622003 & 0.064956 \\
\hline 3 & 6.091976 & 0.052919 & 6.091976 & 0.052919 \\
\hline 4 & 4.816728 & 0.066921 & 4.816728 & 0.066921 \\
\hline 5 & 3.413293 & 0.083014 & 3.403136 & 0.083014 \\
\hline 6 & 3.88459 & 0.094597 & 3.88459 & 0.094597 \\
\hline 7 & 4.329712 & 0.10134 & 4.329712 & 0.10134 \\
\hline 8 & 4.362527 & 0.10134 & 4.362527 & 0.10134 \\
\hline 9 & 4.209553 & 0.099592 & 4.209553 & 0.099592 \\
\hline 10 & 4.146316 & 0.099592 & 4.146316 & 0.099592 \\
\hline 11 & 4.016491 & 0.097715 & 4.016491 & 0.097886 \\
\hline 12 & 3.932913 & 0.096221 & 3.932913 & 0.096221 \\
\hline 13 & 3.850276 & 0.094597 & 3.850276 & 0.094597 \\
\hline 14 & 3.764122 & 0.092848 & 3.764122 & 0.093012 \\
\hline 15 & 3.765768 & 0.093012 & 3.765768 & 0.093012 \\
\hline 16 & 3.86451 & 0.094597 & 3.868813 & 0.094597 \\
\hline 17 & 4.272736 & 0.10134 & 4.272736 & 0.10134 \\
\hline 18 & 5.085253 & 0.110713 & 5.085253 & 0.110713 \\
\hline 19 & 5.024076 & 0.110713 & 5.024076 & 0.110713 \\
\hline 20 & 4.493554 & 0.10496 & 4.493554 & 0.10496 \\
\hline 21 & 4.051834 & 0.097886 & 4.051834 & 0.097886 \\
\hline 22 & 3.577483 & 0.088496 & 3.577483 & 0.088496 \\
\hline 23 & 3.378919 & 0.06463 & 3.378919 & 0.067197 \\
\hline 24 & 4.816728 & 0.044921 & 4.816728 & 0.046758 \\
\hline
\end{tabular}

Table 3. IEEE 24-bus RTS operation cost and security comparison.

\begin{tabular}{llc}
\hline \multicolumn{1}{c}{ Scenarios } & Operation cost $(\$)$ & Number \\
\hline Scenario 1 contingencies \\
Scenario 2 (security + no ESS) & 798934.5488 & - \\
Scenario 3 (no security + ESS) & 761336.2647 & 387 \\
Scenario 4 (security + ESS) & 798765.6080 & - \\
\hline
\end{tabular}

of the system operation in a scenario. Table 2 shows how security considerations can reduce the amount of risk in the operation of the PJM 5-bus test system. In this table, the hourly $P I_{M W}$ is given to compare it variations against each other.

As is obvious, after comparing the abovecalculated $P I_{M W}$ between scenarios 1 and 2 as well as scenarios 3 and 4 , consideration of security constraints reduces the amount of $P I_{M W}$. Concentrating on scenarios 2 and 4 shows that when ESS units discharge at hours $11,14,23$, and 24, the line flows get slightly higher.

\subsection{Case study 2: IEEE 24-bus RTS}

The IEEE 24-bus RTS system characteristics are similar to those in the referenced study [27] and 6 wind generations are added to the system, as given in [28], at buses $3,5,7,16,21$, and 23. All wind generators have a $70 \mathrm{MW}$ generation capacity. Also, in this paper, 6 ESS units with 7 MWh capacity are added to every bus with wind turbines. The charging/discharging efficiency of ESS units is $95 \%$ and $90 \%$, respectively. WVC and VOLL are similar to those in Case 1. The scenarios are illustrated for IEEE 24-bus RTS in Table 3.

The number of binding contingencies is reduced by $87 \%$ and the cost of security from scenario 2 to scenario 4 is reduced by $143.34 \$$ for operation in a 24 $\mathrm{h}$ period through the employment of ESS units. The cost of security in scenarios 2 and 4 is $37572.6833 \$$ and $37429.3433 \$$, respectively.

Figures 4 and 5 illustrate the SOC (MW) and charge/discharge power (MW) of ESS units in the 24-h period of operation, respectively. As in PJM 5-bus test system, the ESS units will charge and discharge during the off-peak and peak times and also, when the related wind turbine is not curtailing and when it is curtailing the generation, respectively. It is obvious that $\operatorname{ESS} 5$ is not dispatched due to the absence of any load on the 


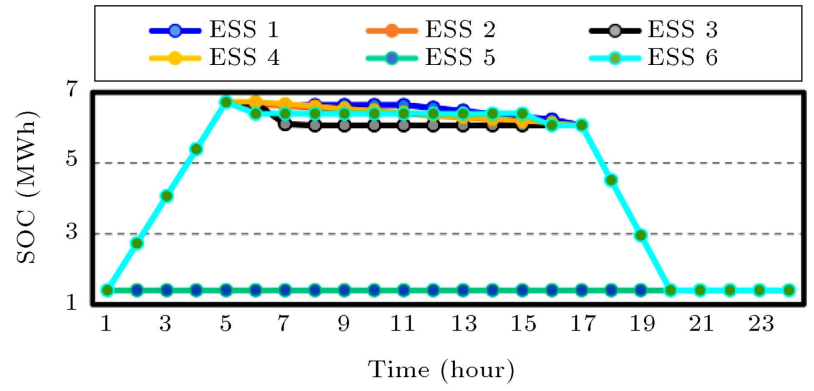

Figure 4. SOC of ESS units for IEEE 24-bus RTS.

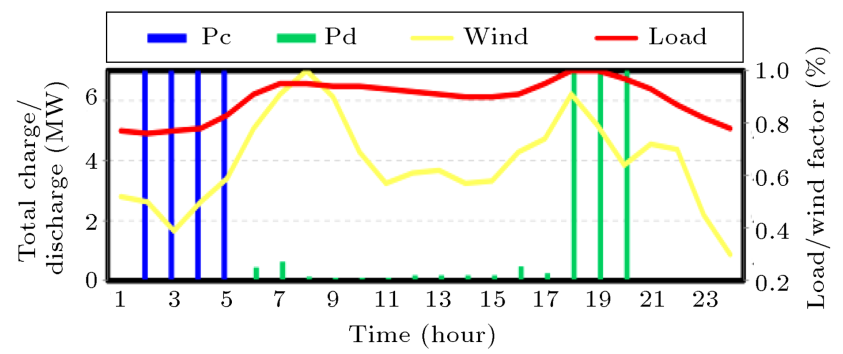

Figure 5. Total charge/discharge power of ESS units for IEEE 24-bus RTS.

bus where ESS 5 lies and the cheapest generation unit there.

In both cases, there is no wind curtailment and load shedding, because the wind generation cost is zero and wind curtailment has a penalty and also when considering the security constraints lines does not hit their limits. In the case of load shedding, according to the sufficient generation of the test systems, there is no need for load shedding.

Similar to Case 1 , the performance index is given in Table 4 to demonstrate how security considerations can help improve the risk management in a power system.

Upon comparing the above-calculated $P I_{M W}$ between scenarios 1 and 2 as well as scenarios 3 and 4, consideration of security constraints reduces the amount of $P I_{M W}$. According to scenarios 2 and 4, it is shown that when ESS units being discharged at hours 6-20, the line flows get slightly higher.

\subsection{Load scale manipulation}

According to the references containing the test systems, the load scales in the base case of PJM 5-bus test system and IEEE 24-bus RTS are close to 0.5 and 0.75 , respectively. Therefore, in order to assess the security of the systems, the load scale is manipulated as follows and results are given in Tables $5-8$ and Figures $6-9$ :

\section{PJM 5-bus test system}

- Load scale: 0.75

- Load scale: 0.95

If there is no ESS in the system when load scale is

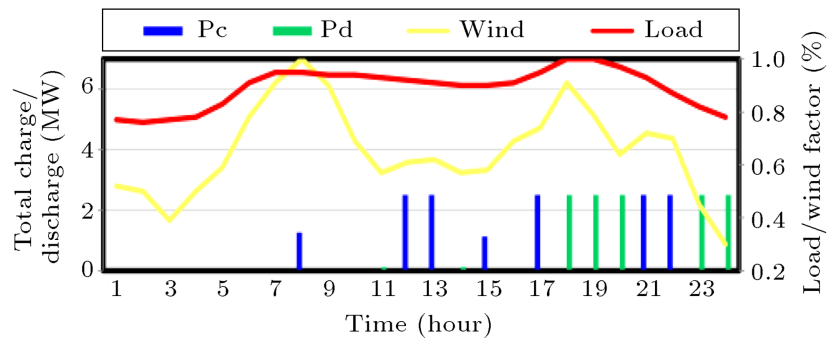

Figure 6. Total charge/discharge power of ESS units for PJM 5-bus test system on 0.75 load scale.

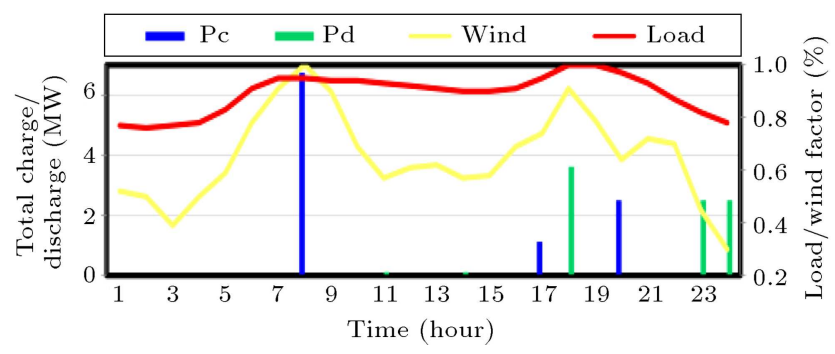

Figure 7. Total charge/discharge power of ESS units for PJM 5-bus test system on 0.95 load scale.

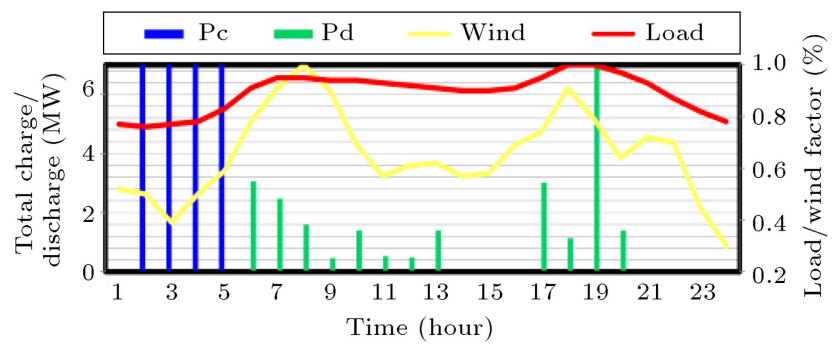

Figure 8. Total charge/discharge power of ESS units for IEEE 24-bus RTS on 0.9 load scale.

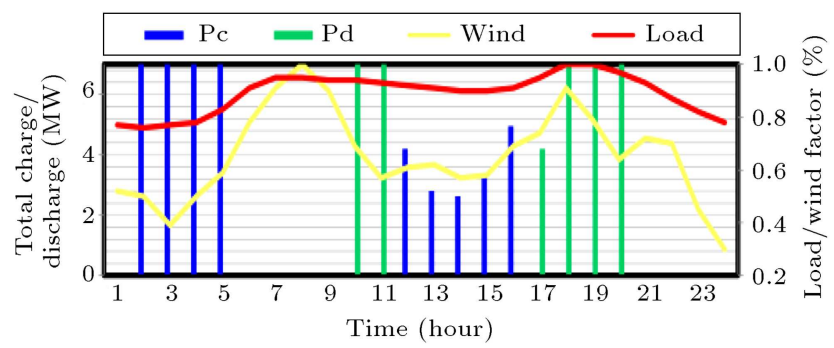

Figure 9. Total charge/discharge power of ESS units for IEEE 24-bus RTS on 0.98 load scale.

greater than 0.75 , the problem is infeasible. However, the presence of the ESS units makes the problem feasible despite the large amount of load shedding.

\section{PJM IEEE 24-bus RTS}

- Load scale: 0.8

- Load scale: 0.98

In this case study, the system can endure $100 \%$ 
Table 4. IEEE 24-bus RTS $P I_{M W}$ amount for each scenario.

\begin{tabular}{|c|c|c|c|c|}
\hline & $P I_{M W}($ sce. 1) & $P I_{M W}$ (sce. 2) & $P I_{M W}($ sce. 3$)$ & $P I_{M W}$ (sce. 4) \\
\hline 1 & 0.033262 & 0.012848 & 0.033262 & 0.012848 \\
\hline 2 & 0.309456 & 0.023664 & 0.303363 & 0.024184 \\
\hline 3 & 0.264072 & 0.03081 & 0.259019 & 0.031544 \\
\hline 4 & 0.274988 & 0.031236 & 0.27122 & 0.03194 \\
\hline 5 & 0.456938 & 0.057714 & 0.448024 & 0.059023 \\
\hline 6 & 0.506998 & 0.045753 & 0.506998 & 0.045943 \\
\hline 7 & 0.594842 & 0.038816 & 0.594842 & 0.038873 \\
\hline 8 & 0.592553 & 0.048292 & 0.592553 & 0.04834 \\
\hline 9 & 0.568264 & 0.042405 & 0.568264 & 0.042441 \\
\hline 10 & 0.582728 & 0.028034 & 0.582728 & 0.028053 \\
\hline 11 & 0.565786 & 0.025226 & 0.565786 & 0.025242 \\
\hline 12 & 0.530566 & 0.028566 & 0.530566 & 0.028595 \\
\hline 13 & 0.505812 & 0.031634 & 0.505812 & 0.031668 \\
\hline 14 & 0.486932 & 0.03157 & 0.486932 & 0.031604 \\
\hline 15 & 0.486747 & 0.032191 & 0.486747 & 0.032226 \\
\hline 16 & 0.504677 & 0.036583 & 0.504677 & 0.035682 \\
\hline 17 & 0.611476 & 0.028152 & 0.611476 & 0.028187 \\
\hline 18 & 0.678136 & 0.02533 & 0.720525 & 0.026303 \\
\hline 19 & 0.49123 & 0.020637 & 0.516026 & 0.021327 \\
\hline 20 & 0.492093 & 0.019504 & 0.517727 & 0.020232 \\
\hline 21 & 0.549339 & 0.031461 & 0.549339 & 0.031461 \\
\hline 22 & 0.461358 & 0.068356 & 0.462951 & 0.069209 \\
\hline 23 & 0.425005 & 0.058708 & 0.42662 & 0.058708 \\
\hline 24 & 0.28181 & 0.039617 & 0.25355 & 0.039617 \\
\hline
\end{tabular}

Table 5. PJM 5-bus test system operation cost and security comparison.

\begin{tabular}{lccc}
\hline \multicolumn{1}{c}{ Scenarios } & Operation cost $(\$)$ & $\begin{array}{c}\text { Number of } \\
\text { contingencies }\end{array}$ & $\begin{array}{c}\text { Total load } \\
\text { shedding (MW) }\end{array}$ \\
\hline Scenario 1 (no security + no ESS) & 482535.0130 & - & 79.5 \\
Scenario 2 (security + no ESS) & 650590.0105 & 672 & 349.197 \\
Scenario 3 (no security + ESS) & 482535.0130 & - & 79.5 \\
Scenario 4 (security + ESS) & 650254.6309 & 276 & 347.957 \\
\hline
\end{tabular}

Table 6. PJM 5-bus test system operation cost and security comparison.

\begin{tabular}{llcc}
\hline \multicolumn{1}{c}{ Scenarios } & Operation cost $(\$)$ & $\begin{array}{c}\text { Number of } \\
\text { contingencies }\end{array}$ & $\begin{array}{c}\text { Total load } \\
\text { shedding (MW) }\end{array}$ \\
\hline Scenario 1 (no security + no ESS) & Infeasible & - & - \\
Scenario 2 (security + no ESS) & Infeasible & - & - \\
Scenario 3 (no security + ESS) & 1037233.8768 & - & 1527.016 \\
Scenario 4 (security + ESS) & 2162297.8581 & 264 & 5713 \\
\hline
\end{tabular}


Table 7. IEEE 24-bus RTS operation cost and security comparison.

\begin{tabular}{lccc}
\hline \multicolumn{1}{c}{ Scenarios } & Operation cost $(\$)$ & $\begin{array}{c}\text { Number of } \\
\text { contingencies }\end{array}$ & $\begin{array}{c}\text { Total load } \\
\text { shedding (MW) }\end{array}$ \\
\hline Scenario 1 (no security + no ESS) & 811638.7398 & - & 0 \\
Scenario 2 (security + no ESS) & 861851.0572 & 409 & 0 \\
Scenario 3 (no security + ESS) & 811546.1912 & - & 0 \\
Scenario 4 (security + ESS) & 861711.4492 & 63 & 0 \\
\hline
\end{tabular}

Table 8. IEEE 24-bus RTS operation cost and security comparison.

\begin{tabular}{lccc}
\hline \multicolumn{1}{c}{ Scenarios } & Operation cost $(\$)$ & $\begin{array}{c}\text { Number of } \\
\text { contingencies }\end{array}$ & $\begin{array}{c}\text { Total load } \\
\text { shedding (MW) }\end{array}$ \\
\hline Scenario 1 (no security + no ESS) & 1107265.7058 & - & 156.719 \\
Scenario 2 (security + no ESS) & 1248705.7896 & 354 & 505.092 \\
Scenario 3 (no security + ESS) & 1107075.2795 & - & 156.227 \\
Scenario 4 (security + ESS) & 1243935.3552 & 26 & 405.424 \\
\hline
\end{tabular}

load scale with some load shedding, but the problem is not infeasible.

\section{Conclusion}

This paper concentrated on the impact of Energy Storage System (ESS) on the security of the power system with high wind penetration. Presence of ESS changed the problem from NLP to an MINLP problem. According to the results obtained in the simulations, the presence of ESS in the power system reduced the security cost by $0.2 \%$ in the PJM 5 -bus test system on 0.75 load scale and $3.2 \%$ in the IEEE 24-bus RTS on 0.98 load scale. Implementation of ESS units also mitigated the number of critical contingencies by $59 \%$ in the PJM 5-bus test system on 0.75 load scale and $93 \%$ in the IEEE 24-bus RTS on 0.98 load scale. Furthermore, results illustrated that ESS units would charge during the off-peak times and discharge at peak times. This method for dispatching the ESS units reduced the contingencies imposed on the system by wind generation unavailability. Also, based on the comparative results obtained from case studies, it can be inferred that the larger the system, the greater the impact of ESS presence on security of the system with high renewable generation penetration.

As a future work, the problem can be modeled in a decentralized fashion to make the regional system management possible. Also, the uncertainties of the wind generations are modeled by probabilistic functions.

\section{Nomenclature}

\section{Sets and indices}

$g \quad$ Index of thermal generating units

$i, j, n, m \quad$ Index of network buses

$\begin{array}{ll}r e f & \text { Reference or slack bus } \\ t & \text { Index of time intervals } \\ \Omega_{G} & \text { Set of thermal generating units } \\ \Omega_{G}^{i} & \text { Set of thermal generating units } \\ & \text { connected to bus } i \\ \Omega_{l} & \text { Set of network branches } \\ \Omega_{l}^{i} & \text { Set of branches connected to bus } i\end{array}$

\section{Parameters}

$L_{i, t} \quad$ Power demand in bus $i$ at time interval

$a_{g}, b_{g}, c_{g} \quad$ Cost function coefficients of thermal unit $g$

$x_{i j} \quad$ Reactance of the branch connecting buses $i$ and $j$

PTDF Power Transfer Distribution Factor

LODF Line Outage Distribution Factor

$P_{i j}^{\max } \quad$ Maximum power flow limit of branch connecting bus $i$ to bus $j$

$P_{g}^{\min / \max } \quad$ Minimum/maximum capacity of thermal generating unit $g$

$R U_{g} \quad$ Maximum ramp up rate of thermal generating unit $g$

$R D_{g} \quad$ Maximum ramp down rate of thermal generating unit $g$

VOLL Value Of Loss Load

VWC Value of Wind Curtailment

$\Lambda_{i}^{w} \quad$ Capacity of wind turbine connected to bus $i$

$\eta_{c} \quad$ Charging efficiency of ESS units

$\eta_{d} \quad$ Discharging efficiency of ESS units

$P_{i, \min / \max }^{c}$ Minimum/maximum charging rate of ESS units 


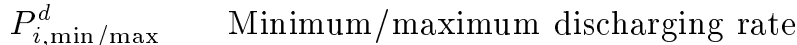
of ESS units

$S O C_{i, \min / \max }$ Minimum/maximum state of charge of ESS units

$\Delta t \quad$ Time interval duration

$X_{i j} \quad$ Element of row $i$ and column $j$ from inverse of network reactance matrix

$w_{i, t} \quad$ Availability of wind turbine connected to bus $i$ at time interval $t$

$P I_{M W} \quad$ Performance index of lines, containing all line flows normalized by their flow limits

$W_{i j} \quad$ Real nonnegative weighting factor to introduce the impact of a line on the performance of the system. Here it is considered equal to 1.

$n \quad$ Exponent of penalty factor

\section{Variables}

$O F$

$P_{g, t}$

$L S_{i, t}$

$P_{i, t}^{w}$

$P_{i, t}^{w c}$

$P_{i, t}^{c}$

$P_{i, t}^{d}$

$P_{i j, t}$

$\lambda_{i, t}$

$\delta_{i, t}$

$S O C_{i, t}$

$\gamma_{i, j, t}$

$U_{i, t}^{c / d}$

\section{References}

1. Yazdaninejadi, A., Hamidi, A., Golshannavaz, S., et al. "Impact of inverter-based DERs integration on protection, control, operation, and planning of electrical distribution grids", Electr. J., 32(6), pp. 43-56 (2019). DOI: $10.1016 /$ j.tej.2019.05.016.
2. Y. Nejadi, A., Sattarpour, T., and Farsadi, M. "Simultaneously optimal placement and operation scheduling of BESSS and DGs in distribution networks in order to minimizing net present value related to power losses", ELECO 2015 - 9th International Conference on Electrical and Electronics Engineering, 16, pp. 434439 (2016).

3. Mohammadi, J., Hug, G., and Kar, S. "Agent-based distributed security constrained optimal power flow", IEEE Trans. Smart Grid, 9(2), pp. 1118-1130 (2018). DOI: $10.1109 /$ TSG.2016.2577684.

4. Bhaskar, M.M. "Security constraint optimal power flow ( SCOPF ) - A comprehensive survey", Trans. Power Syst. , Proterction Distrib., 2, p. 10 (2011). DOI: $10.5120 / 1583-2122$.

5. Alsac, O. and Stott, B. "Optimal load flow with steady-state security", IEEE Trans. Power Appar. Syst., PAS-93(3), pp. 745-751 (1974). DOI: 10.1109/TPAS.1974.293972.

6. Rahmani, S. and Amjady, N. "Improved normalised normal constraint method to solve multi-objective optimal power flow problem", 12, pp. 859-872 (2018). DOI: 10.1049 /iet-gtd.2017.0289.

7. Won, J.R. and Choi, K. "Security-constrained optimal power flow using first-order contingency sensitivity matrix", IFAC Proc. Vol., 36(20), pp. 1019-1023 (2003). DOI: $10.1016 / \mathrm{S} 1474-6670(17) 34608-6$.

8. Capitanescu, F., Glavic, M., Ernst, D., et al. "Applications of security-constrained optimal power flows", Mod. Electr. Power Syst. Symp. MEPS06, September, p. 7 (2006).

9. Phan, D.T. and Kalagnanam, J.R. "Some efficient optimization methods for solving the securityconstrained optimal power flow problem", IEEE Trans. Power Syst., 29(2), pp. 863-872 (2014). DOI: 10.1109/TPWRS.2013.2283175.

10. Dias, L.G. and El-Hawary, M.E. "Security-constrained Opf: influence of fixed tap transformer fed loads", IEEE Trans. Power Syst., 6(4), pp. 1366-1372 (1991). DOI: $10.1109 / 59.116977$.

11. Farsadi, M., Sattarpour, T., and Y. Nejadi, A. "Optimal placement and operation of BESS in a distribution network considering the net present value of energy losses cost", ELECO 2015 - 9th Int. Conf. Electr. Electron. Eng., pp. 434-439 (2016). DOI: 10.1109/ELECO.2015.7394582.

12. Shahidehpour, M. and Fotuhi-friuzabad, M. "Grid modernization for enhancing the resilience, reliability, economics, sustainability, and security of electricity grid in an uncertain environment", 23, pp. 2862-2873 (2016).

13. Prasanta, P., Jain, P., Sharma, S., and Bhaker, R. "Security constrained unit commitment in a power system based on battery energy storage with high wind penetration", 2018 Int. Conf. Power, Instrumentation, Control Comput., pp. 1-6 (2018). 
14. Castillo, A., Gayme, D.F., and Member, S. "Evaluating the effects of real power losses in optimal power flow", 5870(c), pp. 1-13 (2017). DOI: 10.1109/TCNS.2017.2687819.

15. Jannati, J., Yazdaninejadi, A., and Talavat, V. "Simultaneous planning of renewable/ non-renewable distributed generation units and energy storage systems in distribution networks", Trans. Electr. Electron. Mater., 18(2), pp. 111-118 (2017). DOI: 10.4313/TEEM.2017.18.2.111.

16. Thomas, J.J. and Grijalva, S. "Flexible securityconstrained optimal power flow", IEEE Trans. Power Syst., 30(3), pp. 1195-1202 (2015). DOI: 10.1109/TPWRS.2014.2345753.

17. Wen, Y., Guo, C., Kirschen, D.S., et al. "Enhanced security-constrained OPF with distributed battery energy storage", IEEE Trans. Power Syst., 30(1), pp. 98-108 (2015). DOI: 10.1109/TPWRS.2014.2321181.

18. Platbrood, L., Capitanescu, F., Merckx, C., et al. "A generic approach for solving nonlinear-discrete security-constrained optimal power flow problems in large-scale systems", IEEE Trans. Power Syst., 29(3), pp. 1194-1203 (2014). DOI: 10.1109/TPWRS.2013.2289990.

19. Simab, M., Chatrsimab, S., Yazdi, S., et al. "Using integrated method to rank the power system contingency", 24, pp. 1373-1383 (2017).

20. De Quevedo, P. and Contreras, J. "Optimal placement of energy storage and wind power under uncertainty", Energies, 9(7), p. 528 (2016). DOI: 10.3390/en9070528

21. Shim, J.W., Kim, H., and Hur, K. "Incorporating state-of-charge balancing into the control of energy storage Systems for smoothing renewable intermittency", Energies, 12(7), p. 1190 (2019). DOI: 10.3390/en12071190

22. Wood, A.J., Wollenberg, B.F., and Gerald, S.B., Power Generation, Operation and Control, Wiley Inc., Third Ed. (2014).

23. Biskas, P.N. and Bakirtzis, A.G. "Decentralised security constrained DC-OPF of interconnected power systems", Generation, Transmission, and Distribution, IEE Proceedings, 151(6), pp. 747-754 (2004). DOI: 10.1049/ip-gtd:20041063

24. Nangrani, S.P., Power System Security Assessment using Ranking Based on Combined MW-Chaotic Performance Index, IEEE TENCON, Macau, China (2015).

25. "Newyork independent system operator." [Online]. Available: https://www.nyiso.com/.

26. Li, F. and Bo, R. "DCOPF-based LMP simulation: Algorithm, comparison with ACOPF, and sensitivity", IEEE Trans. Power Syst., 22(4), pp. 1475-1485 (2007). DOI: 10.1109/TPWRS.2007.907924

27. González, M., et al., "General rights. An updated version of the IEEE RTS 24-bus system for electricity market and power system operation studies. An updated version of the IEEE RTS 24-bus system for electricity market and power system operation studies", Downloaded from orbit.dtu.dk, pp. 2-7 (2021).
28. Pessanha, J.F.M. "Impact of wind speed correlations on probabilistic power flow by using the Nataf transformation", 2018 IEEE Int. Conf. Probabilistic Methods Appl. to Power Syst., pp. 1-6 (2018). DOI: 10.1109/PMAPS.2018.8440221

\section{Biographies}

Hossein Ebrahimi received the BSc and MSc degrees in Electrical Engineering from Urmia University, Urmia and University of Tabriz, Tabriz, Iran in 2013 and 2019, respectively. He is currently pursuing $\mathrm{PhD}$ degree at the School of Electrical Engineering, Urmia University, Urmia, Iran. His research interests include energy management and power system security.

Mehdi Abapour received the BSc and MSc degrees in Electrical Engineering from the University of Tabriz, Tabriz, Iran in 2005 and 2007, respectively, and the $\mathrm{PhD}$ degree in Electrical Engineering from The Tarbiat Modares University, Tehran, Iran in 2013. Currently, he is an Assistant Professor at the School of Electrical and Computer Engineering, University of Tabriz. His research interests include reliability, energy management, and power electronics.

Behnam Mohammadi Ivatloo received the BSc degree in Electrical Engineering from University of Tabriz, Tabriz, Iran in 2006 and the MSc and PhD degrees from Sharif University of Technology, Tehran, Iran in 2008, all with honors. He is currently an Associate Professor at the Faculty of Electrical and Computer Engineering, University of Tabriz, Tabriz, Iran. His main areas of research are economics, operation, and planning of intelligent energy systems in a competitive market environment.

Sajjad Golshannavaz received the BSc (Honors) and MSc (Honors) degrees in Electrical Engineering from Urmia University, Urmia, Iran in 2009 and 2011, respectively. He received his $\mathrm{PhD}$ degree in Electrical Power Engineering from School of Electrical and Computer Engineering, University of Tehran, Tehran, Iran in 2015. Currently, he is an Assistant Professor at Electrical Engineering Department, Urmia University, Urmia, Iran. Since 2014, he has been collaborating with the smart electric grid research laboratory, Department of Industrial Engineering, University of Salerno, Salerno, Italy. His research interests lie in smart distribution grid operation and planning studies, design of Distribution Management System (DMS), Demand Side Management (DSM) concepts and applications, microgrid design and operation studies, design of Energy Management System (EMS), application of FACTS Controllers in power systems, and application of intelligent controllers to power systems. 